\title{
Analyse des stratégies d'apprentissage dans une méthode d'apprentissage par problèmes : le cas d'étudiantes en soins infirmiers
}

Caroline Larue et Mohamed Hrimech

\section{CpenEdition \\ Journals}

Édition électronique

URL : http://journals.openedition.org/ripes/221

ISSN : 2076-8427

Éditeur

Association internationale de pédagogie universitaire

\section{Référence électronique}

Caroline Larue et Mohamed Hrimech, « Analyse des stratégies d'apprentissage dans une méthode

d'apprentissage par problèmes : le cas d'étudiantes en soins infirmiers », Revue internationale de pédagogie de l'enseignement supérieur [En ligne], 25(2) | 2009, mis en ligne le 14 septembre 2009, consulté le 07 septembre 2020. URL : http://journals.openedition.org/ripes/221 


\title{
Analyse des stratégies d'apprentissage dans une méthode d'apprentissage par problèmes : le cas d'étudiantes en soins infirmiers
}

\author{
Caroline Larue et Mohamed Hrimech
}

\section{L'adoption de l'approche par compétences et de l'apprentissage par problèmes comme véhicule pédagogique}

1 Au Québec, la formation technique en soins infirmiers s'effectue dans les collèges d'enseignement général et professionnel (Cégep). Ceux-ci sont fréquentés par les étudiants qui ont terminé l'équivalent d'études secondaires ( 5 ans) et qui se destinent à des études techniques ou universitaires. À l'instigation du ministère de l'Éducation, du Loisir et des Sports, le programme provincial de formation en soins infirmiers a été revu selon une approche par compétences en 2001. Ce nouveau programme ajoutait à la formation antérieure les soins aux clientèles dans la communauté et les soins dans un contexte ambulatoire faisant écho aux demandes d'un milieu de soins de plus en plus complexes et exigeants envers ses infirmières débutantes.

2 Ce changement a suscité une réflexion des enseignantes sur les méthodes les plus efficaces pour faire acquérir des savoirs complexes tout en poursuivant des objectifs de réussite. À l'automne 2001, les enseignantes du cégep du Vieux-Montréal (ci-après, CVM) ont implanté la méthode d'apprentissage par problèmes (ci-après, APP) comme mode exclusif de l'enseignement des connaissances déclaratives en postulant que les étudiantes seraient incitées à développer des stratégies favorisant la rétention et l'intégration des connaissances, à accroître leur autonomie ainsi qu'à renforcer leurs habiletés de coopération. Mais qu'en est-il vraiment? 
Certaines recherches en APP portent sur le processus d'apprentissage (Aspergen, Bomqvist \& Borgstrom, 1998; Evensen, 2000; Morales-Mann \& Kaitell, 2001; SavinBaden, 2000; White, Amos \& Kouzekanani, 1999)et l'impact du groupe (Duek, 2000 ; Myers \& Horst, 2000)dans une perspective phénoménologique ou ethnographique. La majeure partie des recherches comparent différentes variables tels le raisonnement clinique, la motivation et la réussite, l'intérêt et la participation, l'entraide, le recours aux stratégies d'apprentissage selon que la méthode d'enseignement est en APP ou conventionnelle. Ces recherches rapportent, entre autres, qu'avec la méthode d'APP, les étudiants sont plus précis lorsqu'ils émettent une hypothèse (Hmelo, 1998), ont un meilleur raisonnement clinique (Blumberg, 2000), sont plus habiles à utiliser leurs connaissances en temps réel (Docchy, Segers, Van den Bosshe \& Gijbels, 2003), ont un intérêt soutenu et utilisent davantage de stratégies de traitement en profondeur de l'information, de stratégies de gestion et de stratégies métacognitives que les étudiants des cohortes traditionnelles (Cossette, McClish \& Ostiguy, 2004). Toutefois, d'autres recherches soutiennent que les devis expérimentaux entre les études ne sont pas comparables (Leclercq \& Van der Cleuton, 1998), ou encore que les résultats obtenus par les étudiants dans une méthode d'APP, au regard du jugement clinique, ne sont pas significatifs lorsqu'ils sont mis en relation avec les résultats obtenus par les étudiants qui étudient dans une méthode conventionnelle (Eck, 2002; Soukini \& Fortier, 1999).

Un examen des écrits a révélé peu d'études sur les stratégies d'apprentissage utilisées par les étudiants dans un cadre pédagogique d'APP, ce qui a motivé les questions de recherche suivantes :

- Quelles sont les stratégies d'apprentissage en surface et en profondeur que les étudiantes rapportent utiliser dans le cadre d'un programme en soins infirmiers?

- Quelle est la fréquence d'utilisation des stratégies en profondeur par rapport à la fréquence des stratégies en surface dans le cadre de tutoriaux?

\subsection{L'apprentissage par problèmes}

5 À l'instar de nombreux auteurs (Barrows, 1980 Cossette, Mc Clish \& Ostiguy, 2004 ; Des Marchais, 1996; Dochy, Segers, Van Den Bossche \& Gijbels, 2003; Evensen, 2000 ; Glasgow, 1997; Savin-Baden, 2000), l'APP est défini comme une approche structurée par des étapes et centrée sur la réactivation des connaissances antérieures et le traitement actif des connaissances par l'étudiant. C'est une méthode pédagogique qui allie à la fois le travail individuel et le travail de groupe à partir de problèmes signifiants, interdisciplinaires et inspirés de contextes réels. Cependant, il existe une grande variabilité dans les modalités d'application de l'APP (Charlin, Mann \& Hansen, 1998 ; Des Marchais, 1996 ; Harden \& Davis, 1998 ; Savin-Baden, 2000) ce qui justifie de décrire le terrain dans lequel s'est réalisée l'étude.

6 Au CVM, les groupes d'APP sont composés de 10 à 12 étudiantes sous la direction d'une tutrice, elle-même professeure experte de la matière. Chaque période de tutorat comprend trois phases telles que montrées dans le tableau 1. Durant la phase de découverte du problème (phase 1), le groupe se rencontre environ 90 minutes, reçoit le problème, trouve les mots-clés, établit des liens entre les indices et tente de formuler des hypothèses pour expliquer ces liens. Durant la phase d'études personnelles (phase 2), chaque étudiante travaille seule de trois à six heures, étudie le problème à partir des objectifs d'apprentissage qui ont été remis à la phase subséquente et produit 
un schéma de concepts. Enfin, durant la phase de validation des connaissances (phase 3), le groupe se réunit à nouveau pour une période de 90 minutes et met en commun les connaissances acquises lors de l'étude individuelle.

Tableau 1 : Étapes de l'APP au Cégep du Vieux-Montréal dans le programme de soins infirmiers, inspirées de Soukini et Fortier (1999).

\begin{tabular}{|c|c|l|}
\hline Modalités & Phases & \multicolumn{1}{c|}{ Descriptions } \\
\hline \multirow{2}{*}{$\begin{array}{c}\text { En petit groupe } \\
(90 \text { minutes })\end{array}$} & $\begin{array}{c}1 \\
\text { Découverte du } \\
\text { problème }\end{array}$ & $\begin{array}{l}\text { Identifier les indices et clarifier les termes } \\
\text { Définir le problème et rédiger la liste des phénomènes à } \\
\text { expliquer } \\
\text { Analyser le problème et proposer des hypothèses } \\
\text { Organiser et prioriser les hypothèses } \\
\text { Clarifier les objectifs d'étude }\end{array}$ \\
\hline $\begin{array}{c}\text { Individuel } \\
(7 \text { heures })\end{array}$ & $\begin{array}{c}2 \\
\text { Etude } \\
\text { personnelle }\end{array}$ & $\begin{array}{l}\text { Collecter toutes les informations nécessaires et réaliser un } \\
\text { schéma de concepts-clés }\end{array}$ \\
\hline $\begin{array}{c}\text { En petit groupe } \\
\text { (90 minutes) }\end{array}$ & $\begin{array}{c}3 \\
\text { Validation des } \\
\text { connaissances }\end{array}$ & $\begin{array}{l}\text { Synthétiser et valider les hypothèses } \\
\text { Evvaluer le tutorat }\end{array}$ \\
\cline { 2 - 3 } & Faire son bilan personnel \\
\hline
\end{tabular}

\subsection{Les stratégies d'apprentissage}

7 Nous avons analysé différentes définitions, objectifs et caractéristiques attribués aux stratégies d'apprentissage (Boulet, Savoie-Zajc \& Chevrier 1996 ; Fayol \& Monteil, 1994 ; Frenay, Noël, Parmentier \& Romainville, 1998; Hrimech, 2000; Romano, 1991; Weinstein \& Hume, 1998). Ces définitions sont issues des travaux menés par des innovateurs tels O'Neil (1978) et Schmeck (1988). Les éléments récurrents nous amènent à proposer une synthèse de la définition des stratégies d'apprentissage. Dans cette étude, le concept de stratégie d'apprentissage retenu correspond aux pensées et comportements des étudiantes qui se traduisent par des procédures, des actions utilisées de manière consciente, intentionnelle, flexible et orientée vers la réussite académique.

$8 \quad$ Les stratégies sont regroupées en quatre catégories : 1) les stratégies cognitives ; 2) les stratégies métacognitives ; 3 ) les stratégies affectives; et 4) les stratégies de gestion. La catégorie des stratégies cognitives comprend des stratégies de mémorisation, d'élaboration et d'organisation des connaissances. La catégorie des stratégies métacognitives comprend des stratégies d'autoévaluation, d'autorégulation et d'autocontrôle. La catégorie des stratégies affectives comprend des stratégies de maintien de la motivation, de la concentration et de la gestion des émotions. La catégorie de gestion comprend des stratégies de gestion du temps, de gestion de l'environnement et de gestion des ressources matérielles et humaines.

9 Les chercheurs, tels Weinstein \& Mayer (1986), Entwistle (1988) se sont demandé si certaines stratégies favorisaient davantage un encodage en profondeur de l'information afin que les indices de rappel soient plus présents lorsque la connaissance serait sollicitée dans une situation complexe de travail. 


\subsection{L'approche en profondeur et l'approche en surface}

10 Marton \& Säljo (1976), Entwistle (1988), Romano (1991) distinguent entre l'apprentissage en profondeur et l'apprentissage de surface. L'apprentissage en profondeur correspond à des comportements où les étudiants font un traitement actif de l'information et utilisent des stratégies d'élaboration et d'organisation plutôt que des stratégies de mémorisation. Les stratégies de soutien au traitement de l'information comme les stratégies métacognitives, affectives ou de gestion pourraient favoriser ou contrer l'une ou l'autre approche. Les travaux de Ramsden (1988), Entwistle (1988), Frenay, Noël, Parmentier \& Romainville (1998) autorisent cette interprétation. Dans une approche en profondeur, les étudiants élaborent et organisent leurs connaissances, éprouvent le besoin de faire du sens avec les informations, ont une forte implication affective et utilisent davantage les ressources pour apprendre. C'est l'inverse lorsqu'ils optent pour une approche d'apprentissage en surface. Les étudiants utilisent des stratégies de mémorisation et de reproduction des connaissances, ont un intérêt instrumental pour la connaissance, posent peu d'actions métacognitives, sont peu engagés affectivement et utilisent minimalement les ressources dont ils disposent.

\section{La méthodologie}

11 Afin d'accéder aux stratégies d'apprentissage que les étudiantes utilisent en APP, des entrevues semi-dirigées ont été conduites auprès de 31 d'entre elles, admises à l'automne 2003, dans le programme de soins infirmiers et sélectionnées au hasard dans deux populations d'étudiantes, celles qui proviennent directement du secondaire (population A) et celles qui ont entrepris auparavant des études collégiales dans un autre programme (population B). Dans les deux cas, ce sont majoritairement des filles (87,1\% pour $12,9 \%$ de garçons) et leur moyenne d'âge est de 22.98 ans avec une concentration entre 17 et 23 ans (61,3\% des étudiantes). Le questionnaire d'entrevue, pré-testé auprès de quatre étudiantes, a permis de rapporter leurs conduites durant les tutoriaux au regard des quatre catégories de stratégies. Les entrevues ont porté sur le même tutorat afin de pouvoir comparer les manières de faire et se sont déroulées dans les jours suivant la fin du tutorat pour que les étudiantes puissent se souvenir plus aisément de leurs stratégies d'apprentissage.

Des paramètres externes ont balisé les entretiens afin de réduire au minimum la perte du souvenir : 1) entretien proche du tutorat ; 2) demande, lors de la prise du rendezvous d'entrevue, de consigner par écrit les étapes du travail individuel ; 3) dépôt lors de l'entrevue des productions associées au tutorat tels les notes de l'étudiante, les livres de référence, le problème du tutorat et le schéma.

13 Pour procéder à l'analyse des données, les entretiens ont été transcrits quasi intégralement et découpés en unité de sens. Une grille de codage a été élaborée à partir des catégories du cadre théorique. Ensuite, pour chacune des catégories d'actions, les actions stratégiques que l'étudiante utilise dans le cadre de l'APP ont été identifiées. Enfin, trois juges se sont rencontrés pour une validation interjuge et sont arrivés à un accord supérieur à $90 \%$. Le codage initial a été repris 10 jours plus tard et nous avons aussi obtenu un accord au dessus de $90 \%$. Rappelons que les stratégies qui ont été définies dans cette étude sont celles qui sont rapportées par les étudiantes. Il est possible qu'elles diffèrent de celles qui pourraient être vérifiées par l'observation. 


\section{Résultats}

\subsection{Les stratégies cognitives rapportées lors du travail de groupe} problème et de la phase de validation des connaissances. L'analyse des unités de sens a fait ressortir quatre types d'actions stratégiques dans la catégorie des stratégies de traitement en surface de l'information. Elles sont résumées dans le tableau 2. D'abord, la sélection des mots-clés soit lorsque l'étudiante souligne automatiquement les termes inconnus, ce qui semble relié aux soins, au médical, à la santé, sans intention précise, un peu à l'aveuglette. Ensuite, la prise de notes pour se rappeler ce qui est dit, enfreignant ainsi la consigne de laisser cette tâche cléricale à l'étudiante qui est désignée pour prendre les notes, afin de participer à l'élaboration des connaissances à la phase 1. L'énonciation des indices qui sont soulignés ou des connaissances qui ont été acquises est une action de rappel tout comme l'écoute des autres qui est utilisée afin de retenir l'information qui provient des autres étudiantes.

des unités de sens a fait ressortir trois types d'actions stratégiques dans la catégorie des stratégies de traitement en profondeur de l'information. D'abord, l'énonciation d'une connaissance antérieure lorsque l'étudiante se réfère par analogie ou en établissant des liens entre la situation présentée et une situation antérieure à ses connaissances. Ensuite, la formulation de questions lorsque l'étudiante, en établissant des relations entre les données, veut clarifier un élément d'information. Enfin, la discussion pour émettre des hypothèses ou discuter des connaissances lorsque l'étudiante reconnaît la construction collective des connaissances. On observe au tableau 2 que ces stratégies d'élaboration sont peu fréquentes.

Tableau $2:$ Nombre de sujets qui déclarent une action cognitive sur un total de 31 étudiantes durant le travail de groupe (phase $1: \mathrm{P} 1$ et phase $3: \mathrm{P} 3$ du tutorat)

\begin{tabular}{|c|c|c|c|}
\hline \multirow{2}{*}{$\begin{array}{l}\text { Catégorie des stratégies } \\
\text { cognitives }\end{array}$} & \multirow{2}{*}{ Actions cognitives } & \multicolumn{2}{|c|}{ Nombre de sujets } \\
\hline & & P1 & P3 \\
\hline \multirow{4}{*}{ Stratégie de mémorisation } & Sélection de mots-clés par écrit ou visuellement & 30 & 4 \\
\hline & Énonciation d'un indice ou d'une connaissance & 27 & 23 \\
\hline & Prise de notes & 16 & 28 \\
\hline & Écoute des autres & 21 & 24 \\
\hline \multirow{3}{*}{ Stratégie d'élaboration } & $\begin{array}{l}\text { Énonciation d'une connaissance antérieure ou d'une } \\
\text { expérience }\end{array}$ & 12 & 3 \\
\hline & Formulation de questions & 16 & 16 \\
\hline & $\begin{array}{l}\text { Discussion pour émettre des hypothèses ou discuter des } \\
\text { connaissances }\end{array}$ & 7 & 7 \\
\hline
\end{tabular}

\subsection{La description des stratégies cognitives rapportées lors du travail individuel}

Ces stratégies sont celles qui ont été identifiées lors de la phase de l'étude personnelle. Le tableau 3 résume les actions cognitives recensées. Lors du travail personnel, une des 
tâches importante et complexe est la production d'un schéma de concepts sur une page en format lettre. Les actions de traitement en surface qui ont été retenues sont la mise en évidence des mots clés par écrit lorsque l'étudiante déclare souligner ou surligner en faisant la lecture, la prise de notes mot-à-mot lorsque l'étudiante écrit textuellement les idées principales des livres de référence.

Les actions de traitement en profondeur qui ont été retenues sont l'annotation sous une forme graphique lorsque l'étudiante ne prend pas de notes linéaires, mais procède directement avec une organisation graphique, la catégorisation des données en fonction du jugement personnel lorsque l'étudiante déclare ajouter des catégories à celles qui étaient prévues dans ces objectifs en fonction de ses champs d'intérêt ou de ce qu'elle trouve important, l'élimination des répétitions de données lorsque l'étudiante regroupe sous une même appellation les mots-clés du texte qui se répètent, la visualisation des éléments à organiser lorsque l'étudiante se fait une image mentale de ce qu'elle veut organiser, l'application de connaissances générales au problème lorsque l'étudiante revient au problème spécifique pour appliquer ces connaissances. Là encore, l'examen des données révèle que les étudiantes utilisent peu de stratégies d'organisation des connaissances.

Tableau 3 : Nombre de sujets qui déclarent une action cognitive sur un total de 31 étudiantes durant le travail individuel (Phase 2 du tutorat)

\begin{tabular}{|c|l|c|}
\hline $\begin{array}{c}\text { Catégories de stratégies } \\
\text { cognitives }\end{array}$ & \multicolumn{1}{|c|}{ Actions cognitives } & $\begin{array}{c}\text { Nombre de } \\
\text { sujets }\end{array}$ \\
\hline Stratégie de mémorisation & Mise en évidence des mots-clés par écrit & 14 \\
& Prise de notes sous la forme d'un résumé écrit & 24 \\
\hline Stratégie d'élaboration & Catégorisation en fonction d'objectifs prévus & 31 \\
\hline Stratégie d'organisation & Prises de notes directement sous une forme graphique & 8 \\
\hline & Catégorisation en fonction du jugement personnel & 11 \\
& Élimination des répétitions & 7 \\
& Visualisation des éléments à organiser & 11 \\
\hline $\begin{array}{c}\text { Stratégie de généralisation } \\
\text { et de discrimination }\end{array}$ & Application des connaissances génêrales au problème & 20 \\
\hline
\end{tabular}

Ces résultats conduisent aux trois constats suivants. Premièrement, les étudiantes n'adoptent pas spontanément des stratégies favorisant un traitement en profondeur de l'information malgré les consignes des enseignantes. On songe ici au fait que les étudiantes prennent des notes en phase 1 du tutorat et discutent peu. Deuxièmement, les étudiantes utilisent davantage de stratégies de mémorisation que de stratégies d'élaboration et d'organisation des connaissances. Troisièmement, plus d'étudiantes identifient des stratégies durant le travail individuel que durant le travail de groupe.

\subsection{Les stratégies métacognitives, affectives et de gestion rapportées lors du travail de groupe et lors du travail individuel}

\subsubsection{Les stratégies métacognitives}

19 Pour ce qui est des stratégies métacognitives, trois constats s'imposent. Plus d'étudiantes énoncent des jugements sur leur manière d'apprendre durant le travail individuel que durant le travail de groupe. Les étudiantes font des liens entre les difficultés cognitives identifiées (énoncer des indices, poser des questions) et les 
changements qu'elles veulent effectuer (s'impliquer davantage) lors du travail de groupe. Étrangement, les étudiantes énoncent peu de liens entre les difficultés cognitives identifiées (difficulté à faire des liens) et les changements qu'elles veulent effectuer lors du travail individuel (augmenter le temps d'études).

\subsubsection{Les stratégies affectives}

Pour ce qui est des stratégies affectives, les étudiantes indiquent que leur implication facilite le maintien de leur intérêt et leur concentration lors du travail de groupe.

Je m'en viens passionnée, tout m'intéresse. Chez nous, j'en parle à tout le monde.

Sont écoeurés de m'entendre. J'suis vraiment tombée dedans (Florence, $1^{\text {re }}$ session).

Les sujets sont de plus en plus intéressants. Tout m'intéresse. (Valérie, $3^{\mathrm{e}}$ session).

L'intérêt élevé pour les thèmes peut expliquer que peu d'étudiantes ont rapporté des situations générant des émotions suffisamment négatives pour les inciter à développer des stratégies de contrôle. Toutefois, les étudiantes sont attentives à leur état affectif et vont intervenir rapidement pour maintenir la motivation ou prévenir un épuisement. Les actions préventives sont soit temporaires (interrompre le travail ou sortir), soit permanentes (développer de nouvelles habitudes de vie ou une nouvelle manière de penser).

\subsubsection{Les stratégies de gestion}

Enfin, en examinant la catégorie de stratégies de gestion, on constate que la quasitotalité des étudiantes sont ponctuelles lors de la phase 1 et de la phase 3 du tutorat, qu'elles utilisent les livres et le matériel requis, qu'elles débutent leurs travaux à l'avance, étudient à la maison, en silence et réfèrent aux collègues lors de difficultés.

\subsection{Les stratégies en surface ou en profondeur du traitement de l'information}

23 À la suite de cette description des stratégies et de leur fréquence, nous avons examiné les stratégies en surface ou en profondeur de l'apprentissage en tutorat. Rappelons que l'étudiante qui utilise une approche en profondeur de l'apprentissage identifie des actions associées à des stratégies d'élaboration, d'organisation, de généralisation et de discrimination, énonce un engagement personnel et cognitif ainsi qu'une utilisation maximale des ressources matérielles et humaines.

Pour distinguer les actions associées à une approche en profondeur du traitement de l'information au regard de celles associées à une approche en surface, nous avons convenu de dénombrer les actions en profondeur déclarées par les étudiantes pour la catégorie des stratégies cognitives. Nous avons ensuite examiné la proportion d'actions en profondeur déclarée par l'étudiante par rapport à l'ensemble des actions en profondeur.

Pour la catégorie des stratégies affectives, nous sommes retournés aux stratégies de maintien de l'intérêt et avons identifié les étudiantes qui avaient rapporté utiliser l'implication personnelle comme action facilitant le maintien de l'intérêt. La mention d'une implication personnelle de la part de l'étudiante a été retenue comme indicateur d'une approche plus en profondeur de l'apprentissage, peu importe la phase du tutorat. 

investissement cognitif de la part de l'étudiante à partir de deux indicateurs: l'évaluation de son travail à partir de son propre jugement et l'ajout de travail lors de la période d'études individuelle. Les étudiantes mentionnant l'une ou l'autre action sont considérées utiliser une approche en profondeur. Nous sommes arrivés à la conclusion que peu d'étudiantes utilisent une approche en profondeur, car peu d'entre elles déclarent une implication affective, une utilisation maximale des ressources et des stratégies d'élaboration et d'organisation des connaissances. En effet, le tableau 4 montre, sur le plan des stratégies cognitives, que les étudiantes sont plus nombreuses à rapporter des stratégies de mémorisation plutôt que des stratégies d'élaboration ou d'organisation des connaissances.

Tableau 4 : Pourcentage des stratégies de mémorisation et des stratégies d'élaboration ou d'organisation déclarées lors du travail de groupe et du travail individuel

\begin{tabular}{|l|c|c|c|}
\hline \multirow{2}{*}{ Catégorie de stratégies cognitives } & \multicolumn{2}{|c|}{ Travail de groupe } & \multirow{2}{*}{$\begin{array}{c}\text { Travail } \\
\text { individuel }\end{array}$} \\
\cline { 2 - 3 } & Phase 1 & Phase 3 & \\
\hline Stratégies de mémorisation & $77 \%$ & $58 \%$ & $61 \%$ \\
\hline Stratégies d'élaboration ou d'organisation & $39 \%$ & $29 \%$ & $29 \%$ \\
\hline Total & $100 \%$ & $100 \%$ & $100 \%$ \\
\hline
\end{tabular}

\section{Discussion}

À la question: Quelles sont les stratégies que les étudiantes déclarent utiliser, deux constats s'imposent. En premier, les étudiantes font des choix parmi les actions stratégiques qui leurs sont proposées. Bien que les tutrices demandent de ne pas écrire, de se référer à leurs volumes, d'effectuer l'autoévaluation formative ainsi qu'un retour sur les hypothèses en phase 2 et en phase 3 , la majorité des étudiantes ne répondent pas à la demande. Peut-être s'agit-il d'un malentendu pédagogique (Romainville, 1993): les étudiantes sont orientées vers la réussite des examens à court terme alors que les enseignantes veulent orienter les étudiantes vers un apprentissage durable.

Cette constatation pose tout de même la question de l'enseignement des stratégies. Plusieurs chercheurs estiment que l'enseignement des stratégies surtout métacognitives est un facteur de soutien à la réussite (Leamnson, Côté, Bellavance, Chamberland \& Graillon, 2004 ; Romainville, 1993; Weinstein \& Mayer, 1986). Toutefois, il faut aussi, comme le mentionne Entwistle (1988), que les étudiantes soient convaincues des bénéfices qu'elles pourraient tirer de l'utilisation de nouvelles stratégies, en fassent l'expérience et récidivent. Pour que les étudiantes soient convaincues, il faudrait que les enseignantes tiennent compte dans la préparation de 
leurs enseignements de ce qui motive les étudiantes et c'est souvent la réussite de l'examen.

30 À la question : quelle est la part des stratégies qui favorisent une approche en surface ou en profondeur de l'apprentissage, les résultats montrent que les étudiantes utilisent davantage de stratégies de mémorisation que d'élaboration ou d'organisation malgré un contexte d'apprentissage favorable : la méthode d'APP et le contexte de formation professionnelle auquel les étudiantes peuvent s'identifier. Ce résultat va dans le même sens que d'autres obtenus antérieurement notamment par Cartier (1997) et Lahtinen, Lonka \& Lindblom-Ylanne (1997). Nous suggérons deux pistes d'interprétation de ce résultat. La première avance que le développement de nouvelles stratégies nécessite du temps (Cartier, 1997). On se rappellera que ces étudiantes sont à la dixième semaine de la première session et qu'elles manquent de temps pour concilier l'ensemble de leurs activités (étude, travail, famille et loisirs). La deuxième piste pointe en direction du contexte qui peut masquer des pratiques pédagogiques conventionnelles dans la manière d'animer les tutoriaux (Maudsley, 1999).

31 Certains chercheurs proposent de contrôler les paramètres qui favorisent le succès d'une méthode. Ces paramètres sont au nombre de quatre. Le premier, consiste à s'interroger sur l'interprétation et l'importance attribuée à la tâche demandée aux étudiants (Martin \& Briggs, 1986 ; McCombs \& Marzano, 1990 ; Ramsden, 1988 ; Rotter, 1966). Il est possible que certaines étudiantes en APP perçoivent et interprètent la tâche demandée comme étant de la mémorisation nécessitant peu d'engagements cognitif et affectif. Le deuxième est le type de stratégies antérieures acquises (Entwistle, 1988). De nombreuses étudiantes ont mentionné, lors de leurs études antérieures, être davantage préoccupées par le rendement aux examens que par l'intégration des connaissances à plus long terme. Le troisième est le type d'évaluation du cours (Marton \& Säljo, 1976; Ramsden, 1988; Weinstein \& Hume, 1998). Une évaluation de cours proposant des questions à choix de réponses serait plus susceptible de favoriser une étude en surface alors qu'une évaluation proposant des questions à développement induirait davantage une étude en profondeur. Dans le curriculum des étudiantes de cette recherche, l'évaluation est composée de questions ouvertes à réponse courte ce qui devrait les inciter à adopter une approche en profondeur de l'apprentissage. Cependant, cette hypothèse n'a pas été vérifiée dans nos résultats. Le quatrième paramètre, toutefois, concerne les exigences de l'évaluation (Watkin, 1984). Une évaluation qui exige un niveau élevé d'élaboration et d'organisation des connaissances devrait s'appuyer sur une étude en profondeur alors qu'une évaluation qui exige un niveau de restitution des connaissances devrait se fonder sur une étude en surface. L'analyse rapide du questionnaire du premier examen en soins infirmiers relève la présence de questions nécessitant exclusivement des stratégies de mémorisation. Sur ce point, nous partageons l'avis de Leamnson (1999) voulant que les formes d'évaluation des cours déterminent, en partie, les stratégies d'apprentissage qui seront utilisées par les étudiants.

En terminant, examinons les implications de la recherche pour la communauté des chercheurs et les tutrices de l'enseignement en APP. 


\section{Implications de la recherche et limites}

Une des retombées immédiates a été de poursuivre l'étude sur trois sessions afin d'estimer l'évolution de l'adoption de stratégies favorisant une approche en profondeur. Les résultats préliminaires de cette étude pointent vers une plus grande utilisation des stratégies favorisant un apprentissage en profondeur à latroisième session.

D'autres perspectives se dessinent. L'étude des schémas réalisés par les étudiantes permettrait d'évaluer comment celles-ci concourent à l'organisation et au regroupement des connaissances. Sur le plan méthodologique, l'observation des actions des étudiantes en tutorat ajouterait de la validité à leurs déclarations. Une meilleure connaissance de la perception du rôle des tutrices et de leurs activités en tutorat aiderait à mieux cerner les consignes données qui conditionnent l'apprentissage des étudiantes (Larue, 2008). L'étude de la relation entre les formes d'évaluation et le développement de l'approche en profondeur est cruciale et mérite d'être poursuivie de manière prioritaire. Enfin, l'étude du transfert des stratégies en clinique permettrait d'évaluer l'impact de cette méthode sur la performance clinique.

Sur le terrain de l'enseignement, nous discernons trois retombées de la recherche. Premièrement, le soutien du rôle de tutrice doit être effectué sur trois plans. D'abord, il convient de procéder à un ajustement du rôle de tutrice durant le travail de groupe afin que celle-ci utilise davantage les connaissances antérieures des étudiantes lors de la phase un du tutorat, propose une application des connaissances acquises en présentant de nouvelles situations en phase trois, mise sur l'identité professionnelle et l'engagement pour que les connaissances acquises soient directement utilisées en milieu de stage. Ensuite, il est essentiel de fournir du soutien aux tutrices pour qu'elles puissent aider et encadrer les étudiantes à réaliser leur schéma. Une formation à l'enseignement de stratégies complexes par l'élaboration d'un programme de formation initiale et continue aux tutrices s'impose. Cette formation devrait mettre l'accent sur l'art de poser des questions qui suscitent l'utilisation de stratégies cognitives d'élaboration, d'organisation, de généralisation et de discrimination des connaissances. Les tutrices devraient s'exercer à formuler des questions spécifiques qui visent, entre autres, l'élaboration des connaissances: «Que veux-tu dire par cette expression?», «Peux-tu préciser cette affirmation?» ou le regroupement des données: «Est-ce que ces éléments pourraient faire partie d'une catégorie plus large ? ", « S'agit-il d'une même famille de données ? ».

Deuxièmement, bien que l'APP soit un véhicule puissant pour approfondir les connaissances, la méthode repose sur une cohérence entre les problèmes à l'étude, les lectures demandées et l'évaluation. Des mécanismes de suivi pour assurer la meilleure cohérence possible sont à prévoir.

Troisièmement, la formation des étudiantes à l'importance du travail de groupe pour comprendre et résoudre une situation de soins ainsi qu'un dépistage rapide des étudiantes à risque d'échec pourraient être effectués par un sondage sur leur attente de performance et leur intérêt pour les tutoriaux. Ce dépistage serait suivi par une démarche de la conseillère en orientation amenant à consolider ou modifier leur choix vocationnel. 
nfin, il est opportun de rappeler les limites méthodologiques de cette recherche. Les données recueillies sont subjectives, l'étudiante rapporte ce dont elle se souvient et ce dont elle peut avoir pris conscience lors de l'entretien lui-même. Le nombre de sujets et le contexte limité (un seul site) ne permettent pas de généraliser les résultats obtenus à d'autres domaines. La deuxième limite est conceptuelle. La catégorisation des stratégies d'apprentissage est différente selon les chercheurs et nous avons identifié des éléments de chevauchements entre les stratégies affectives, métacognitives et de gestion. Enfin, comme toute recherche qualitative, les résultats sont associés au contexte. Il s'agit, ici, d'étudiantes en soins infirmiers utilisant l'apprentissage par problèmes et il faudrait une réplication de l'étude dans divers contextes pour en tirer des conclusions généralisables. Toutefois, il est fort à parier que certains professeurs reconnaîtront le profil de leurs propres étudiants en APP.

\section{BIBLIOGRAPHIE}

Aspegren, K., Blomqvist, P., \& Borgstrom, A. (1998). Live patients and problem-based learning. Medical Teacher, 20(5), 417-420.

Blumberg, P. (2000). Evaluating the evidence that problem-based learners are self-directed learners : A review of literature. DansD. H. Evensen et C. E. Hmelo (Éds)., Problem-based learning : A research perspective on learning interactions (pp.199-226). Mahwah, NJ: Lawrence Erlbaum Associates, Inc.

Boulet, A., Savoie-Zajc, L. \&, Chevrier, J. (1996). Les stratégies d'apprentissage à l'université. SainteFoy, Qc : Presses de l'Université du Québec.

Cartier, S. (1997). Lire pour apprendre: description des stratégies utilisées par des étudiants en médecine dans un curriculum d'apprentissage par problèmes. Thèse de doctorat inédite, Université de Montréal.

Charlin, B., Mann, K., \& Hansen, P. (1998). The many faces of problem-based learning.Medical Teacher, 20(4),323-330.

Cossette, R., McClish, S., \& Ostiguy, K. (2004). L'apprentissage par problèmes en soins infirmiers. Adaptation en clinique et évaluation de ses effets. Rapport de recherche. Montréal : Cégep du Vieux Montréal.

Côté, D. J., Bellavance, C., Chamberland, M., \& Graillon, A. (2004). Un programme pour aider les étudiants en médecine à développer leurs stratégies d'apprentissage. Pédagogie médicale, 5(2), 95-102.

Des Marchais, J. E. (1996). Apprendre à devenir médecin : bilan d'un changement pédagogique centré sur l'étudiant. Sherbrooke : Presses de l'Université de Sherbrooke. 
Dochy, F., Segers, M., Van den Bossche, P., \& Gijbels, D. (2003). Effects of problem-based learning: a meta-analysis. Learning and Instruction, 13(5), 533-568.

Duek, J.E. (2000). Whose group is it anyway? Equity of student discourse in problem basedlearning ? Dans D. H. Evensen \& C. E. Hmelo (Éds.), Problem-based learning : A research perspective on learning interactions (pp.75-107). Mahwah, NJ : Lawrence Erlbaum Associates, Inc.

Eck, J. C. (2002). Assessing student outcomes in general education at Samford University. Journal of General Education, 51(4), 316-325.

Entwistle, N. (1988). Motivational factors in students' approaches to learning. DansR. R. Schmeck (Éd.), Learning strategies and learning styles (pp.21-51). New York : Plenum Press.

Evensen, D. H. (2000). Observing self directed learners in a problem-based learning context : two case studies. Dans D. H. Evensen \& C. E. Hmelo (Éds.), Problem-based learning. A research perspective on learning interactions (pp.263-297). Mahwah, NJ : Lawrence Erlbaum Associates, Inc.

Fayol, M., \& Monteil, J.M. (1994). Stratégies d'apprentissage et apprentissage des stratégies. Revue française de pédagogie, 106, 91-110.

Frenay, M., Noël, B., Parmentier, P., \& Romainville, M. (1998). L'étudiant-apprenant. Grilles de lecture pour l'enseignant universitaire. Bruxelles: De Boeck.

Hmelo, C. E. (1998). Problem-based learning : Effects on the early acquisition of cognitive skill in medicine. The journal of the learning sciences, 7(2), 173-208.

Hrimech, M. (2000). Les stratégies d'apprentissage en contexte d'autoformation. Dans R. Foucher, \& M. Hrimech (Éds.), L'autoformation de l'enseignement supérieur : apports européens et nordaméricains pour l'an 2000 (pp.99-111). Montréal : Les éditions Nouvelles.

Lahtinen, V., Lonka, K., \& Lindblom-Ylänne, S. (1997). Spontaneous study strategies and the quality of knowledge construction. British Journal of Educational Psychology, 67(1), 13-24.

Larue, C. (2008). Group Learning strategies for nursing students : Reflections on the tutor. International Journal of Nursing Education Scholarship, 5(1), 1-17.

Leamnson, R. N. (1999). Thinking about teaching and learning: developing habits of learning with first year college and university students. Sterling, VA: Stylus Publishing.

Leclercq, D. \& Van der Vleuten, C. (1998). Problem-based learning ou apprentissage par problèmes. DansD. Leclercq (Éd.), Pour une pédagogie universitaire de qualité (pp.189-205). Liège : Mardaga.

Martin, B., \& Briggs, L. (1986). The affective and cognitive domains: integration for instruction and research. Englewoods Cliffs, NJ : Educational Technology Publications.

Marton, F., \& Säljo, R. (1976). Outcome as a function of the learner's conception of the task. British Journal Education of Psychology, 46(2), 115-127.

Maudsley, G. (1999). Do we all mean the same thing by « Problem-based learning »? A review of concepts and a formulation of the ground rules. Academic Medicine, 74(2), 178-185.

McCombs, B. L., \& Marzano, R. J. (1990). Putting the self in self-regulated learning : The self as agent in integrating will and skill. Educational Psychologist, 25(1), 51-69.

Morales-Mann, E.T., \& Kaitell, C.A. (2001). Problem-based learning in a new Canadian curriculum. Journal of Advanced Nursing, 33(1), 13-19. 
Myers, I., \& Horst, S. (2000). Groups in problem-based learning (PBL) : Essential elements in theory and practice. Dans D. H. Evensen \& C. E. Hmelo (Éds.), Problem-based learning : a research perspective on learning interactions (pp.263-297). Mahwah, NJ : Lawrence Erlbaum Associates, Inc.

O’Neil, H. F. (Éd.) (1978). Learning strategies. New York : Academic Press.

Ramsden, P. (1988). Context and strategy : Situational influences on learning. DansR.R. Schmeck (Éd.), Learning strategies and learning styles (pp.159-184). New York : Plenum Press.

Romainville, M. (1993). Savoir parler de ses méthodes : métacognition et performance à l'Université. Bruxelles: De Boeck.

Romano, G. (1991). Étudier... en surface ou en profondeur ? Pédagogie collégiale, 5(2), 6-11.

Rotter, G. E., \& Ashton, D. (1966). Physical education for Nebraska elementary schools. Lincoln, NB : Department of Education, Division of Instructional Services.

Savin-Baden, M. (2000). Problem-based learning in higher education: Untold stories. Buckingham : The Society for Research into Higher Education \& Open University Press.

Schmeck, R.R. (1988). An introduction to strategies and styles of learning. Dans R.R. Schmeck (Éd.). Learning strategies and learning styles (pp.3-19). New York : Plenum Press.

Soukini, M. \& Fortier, J. (1999). Apprentissage par problèmes : étude exploratoire de son application partielle au collégial. Mémoire de maîtrise inédit. Sherbrooke, Qc: Université de Sherbrooke.

Watkins, D. (1984). Students perceptions of factors influencing tertiary learning. Higher Education Research \& Development, 3(1), 33-50.

Weinstein, C.E., \& Hume, L. (1998). Study strategies for lifelong learning. Washington, DC : American Psychological Association.

Weinstein, C. E., \& Mayer, R. E. (1986). The teaching of learning strategies. DansM. C. Wittrock (Éd.), Handbook of research on teaching (pp.315-327). New York : McMillan Publishing Company.

White, M. J., Amos, E., \& Kouzekanani, K. (1999). Problem based-learning : An outcomes study. Nurse Educator, 24(2), 33-36.

\section{NOTES}

1. Pour refléter la prédominance féminine en soins infirmiers autant que par souci d'alléger le texte, le féminin englobe le masculin lorsqu'il est question de la recherche effectuée. Par ailleurs, le masculin est utilisé lorsque les informations ne font pas référence au contexte de l'étude.

\section{RÉSUMÉS}

Plusieurs institutions d'enseignement supérieur ont adopté l'apprentissage par problèmes comme méthode pédagogique, mais peu d'études, à notre connaissance, se sont intéressées à décrire les stratégies utilisées par les étudiants dans un curriculum d'apprentissage par problèmes. Le but de cette recherche est justement l'étude de ces stratégies. L'analyse des 
verbatims obtenus auprès de 31 étudiantes $^{1}$ de soins infirmiers a permis de dégager un ensemble de stratégies et d'en évaluer la fréquence. Les résultats suggèrent que les étudiantes utilisent davantage des stratégies de traitement en surface de l'information que des stratégies de traitement en profondeur malgré des indications voulant que l'utilisation d'une approche en profondeur soit associée à une meilleure réussite au cours.

Several higher education institutions have adopted problem-based learning as an educational method. But, as far as we know, very few studies try to describe the strategies which are used by students in order to succeed in a problem-based learning context. The purpose of this research is to study those strategies. The analysis of the verbatim obtained from 31 nursing students uncovered the main strategies and their respective frequency. The results suggest that the students are more likely to use strategies based on surface treatment of the information than indepth learning strategies. This preference is maintained even though they were told that indepth learning strategies increase their academic achievements.

INDEX

Mots-clés : apprentissage par problèmes, apprentissage en profondeur, stratégies d'apprentissage, travail de groupe, études infirmières

Keywords : problem-based learning, deep learning, learning strategies, group work, nursing studies

\section{AUTEURS}

\section{CAROLINE LARUE}

Professeure adjointe à la Faculté des sciences infirmières de l'Université de Montréal, chercheuse du centre d'innovation en formation infirmière, chercheuse associée au GRIISIQ, chercheuse associée au centre de recherche Fernand-Séguin.

Faculté des sciences infirmières, 2375 chemin de la Côte Sainte-Catherine, Montréal Québec H3T 1A8

Courriel : caroline.larue@umontreal.ca

\section{MOHAMED HRIMECH}

Professeur à la Faculté des sciences de l'éducation de l'Université de Montréal.

Faculté des sciences de l'éducation, 90 Avenue Vincent d'Indy,

Montréal Québec H3T 1A8

Courriel : mohamed.hrimech@umontreal.ca 